\section{MS8-O3 Fidelity of translation: structural view}

Alexey $\operatorname{Rozov}^{1}$, Natalia Demeshkina ${ }^{1}$, Eric Westhof ${ }^{2}$, Marat Yusupov $^{1}$, Gulnara Yusupova ${ }^{1}$

1. Institut de Génétique et de Biologie Moléculaire et Cellulaire, Illkirch, France

2. Institut de Biologie Moléculaire et Cellulaire, Université de Strasbourg, Strasbourg, France

email: rozov@igbmc.fr

mRNA translation by the ribosome is a complex and a highly error-prone process. The ribosome demonstrates very efficient discrimination to fit a right tRNA to mRNA codon. The discrimination of various near-cognate tRNA (with just one mismatch) presents a challenge, since the energy level differences are additionally influenced by the nature of the mismatch and its position in the codon-anticodon duplex. Therefore it is safe to presume that ribosome must utilize some kind of amplifying mechanism to bring the differences between tRNA to an easily discernable level.

First attempts to study the structure of ribosome decoding center were focused on the crystal structure of the $30 \mathrm{~S}$ subunit soaked with short analogs of mRNA and tRNA (1). It was suggested that ribosome undergoes certain conformational changes induced strictly by the binding of cognate tRNA. Recently, our group has reported crystal structures of the $70 \mathrm{~S}$ ribosome carrying cognate and near-cognate tRNAs in the A site (2). The conformational rearrangements of the $30 \mathrm{~S}$ subunit appeared to be identical for all cases and, hence, non-discriminatory. The behavior of the introduced mismatches was, however, surprising. $\mathrm{G} \bullet \mathrm{U}$ base pairs, replacing the canonical $\mathrm{G} \bullet \mathrm{C}$ at either first or second position, adopted Watson-Crick geometry instead of usual wobble, implying involvement of rare tautomeric or ionic forms of the nitrogen bases. This finding led us to propose a new model of the decoding process. It appears that tRNA discrimination is mediated by the rigid mold of the ribosome decoding center which imposes the energy cost of fitting a mismatched base pair into the limits of the standard duplex geometry. Such cost will be high enough for the rejection of near-cognate tRNA in favor of cognate.

Here we present several structures of $70 \mathrm{~S}$ ribosomes co-crystallized with mRNA and tRNAs featuring various mismatches in the first and second position of the A-site and P-site, modeling binding of near-cognate tRNA during decoding. The conclusions derived from our structural investigations are corroborated by the in vivo investigation of mistranslation events (3). Taken together, our data substantiate the proposed model of the rigid decoding center and provide an insight into explanation of variable protein mutation frequency.

1. Ogle JM, et al., Cell, 111(5), 721-732 (2002)

2. Demeshkina N, et al., Nature, 484(7393), 256-259 (2012)

$$
\text { 3. Manickam N, et al., RNA, 20(1), 9-15 (2014) }
$$

Keywords: translation, ribosome structure, X-ray crystallography
MS8-04 Light-induced structural changes in a bacterial phytochrome

Heikki P. Takala ${ }^{1}$

1. University of Jyväskylä, Finland

email: heikki.p.takala@jyu.fi

Phytochromes are light-sensing proteins found in plants and bacteria. They bind a linear tetrapyrrole chromophore (e.g. biliverdin) and cycle between a resting $\mathrm{Pr}$ and an activated Pfr state in response to the light environment. Canonical phytochromes contain a photosensory core region that includes a chromophore-binding domain (CBD) and a phytochrome-associated (PHY) domain. In bacterial phytochromes the photosensory region is followed by an output region that usually consists of a histidine kinase domain.

Our research aims to study the structural changes accompanying the phytochrome photocycle. Here we report light-induced changes in a bacterial phytochrome characterized by the means of X-ray-based methods [1]. SAXS and time-resolved WAXS indicate large light-driven structural changes in phytochrome dimer that occur in millisecond time scale. Most importantly, we have solved seminal crystal structures of phytochrome photosensory core region in its inactive and active states. The structures reveal light-induced refolding close to the chromophore, which leads to opening of the entire phytochrome dimer as the PHY domains move relative to each other (Figure 1). This large-scale opening is linked to the X-ray scattering (S/WAXS) data by molecular dynamics simulations.

These results can be considered as a breakthrough in the phytochrome field. The structural information helps us understand the how these light-driven molecular switches work, and it sets basis for a wealth of applications.

References:[1] Takala H, Björling A, Berntsson O, Lehtivuori H, Niebling S, Hoernke M, Kosheleva I, Henning R, Menzel A, Ihalainen JA, Westenhoff S. (2014) Signal amplification and transduction in phytochrome photosensors. Nature 509(7499):245-8. doi: 10.1038 /nature 13310 .

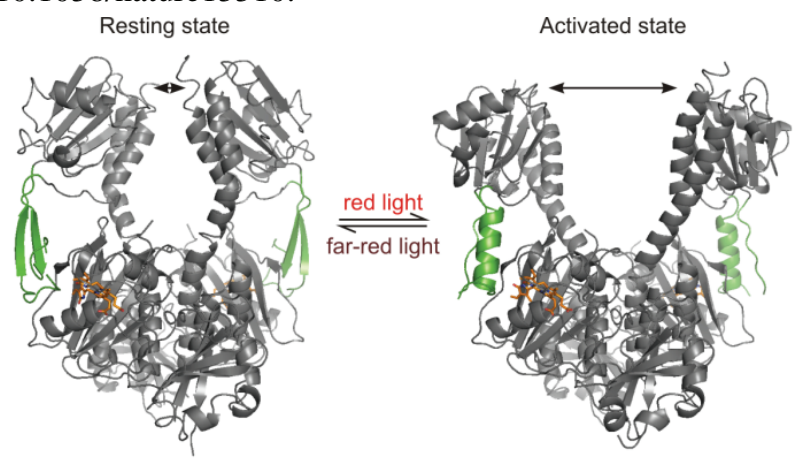

Figure 1. Crystal structures of the photosensory module dimer in the resting (left) and activated (right) states. Upon red light illumination the tongue of the PHY domain (green) changes its fold and the dimer opens up for several nanometers. Figure adapted from [1].

Keywords: phytochrome, photosensor, photocycle, light activation 